\title{
Heatstroke: clinical experience from an Italian ICU and review of the literature
}

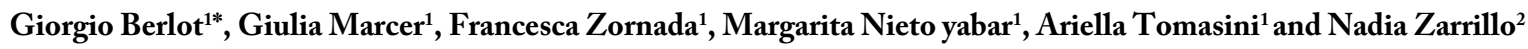 \\ ${ }^{1}$ Department of Anesthesia and Intensive Care, University of Trieste, Italy \\ ${ }^{2}$ Department of Anesthesia and Intensive Care, Caserta Hospital, Italy
}

\begin{abstract}
Objective: to describe the clinical course of patients admitted to our ICU with heat stroke during Summer 2015.

Patients and methods: from July, the 1st to August the 15th nine patients were admitted with the initial diagnosis of septic shock and meningitis; however, subsequent investigations demonstrated that in eight of them ( $5 \mathrm{M}, 4 \mathrm{~F}$, age 76 years, IQR 49-81 years) the symptoms had to be ascribed to a non-exertional heat stroke whereas in the remaining one (M, age 23 years) to an exertional heat stroke. All patients with non-exertional heat stroke presented severe underlying chronic disease and most of them were treated with antipsychotic or antidepressant agents.

Results: in patients with non-exertional heat stroke the clinical course was marked by the elevated body temperature unresponsive to the standard measures associated with the rapid deterioration of the neurologic conditions and an overwhelming multiple-organ dysfunction syndrome; overall, six patients died. The patient with exertional heat-stroke presented only neurologic symptoms that rapidly subsided after cooling.
\end{abstract}

Conclusions: In patients with abnormally elevated body temperature and in the presence of favoring conditions a high index of suspicion for the diagnosis of heat stroke is warranted in order to initiate promptly a life-saving treatment consisting in the rapid cooling of the patients.

\section{Introduction}

A huge number of pathologic processes can be associated with an increase of body temperature (BT) In the Intensive Care Unit (ICU) patients [1]. Although the terms fever, pyrexia and hyperthermia are often used interchangeably, some authors use the the first to indicate a core $\mathrm{BT} \geq 38.3^{\circ} \mathrm{C}$ due to the action of pyrogens on the hypothalamus and the latter two to indicate a $\mathrm{BT} \geq 38.2^{\circ} \mathrm{C}$ determined by causes other than pyrogens, including environmental and pharmacologic factors [2].

Independently from the underlying mechanism, in most circumstances the raised BT represents more a symptom than a disease in and by itself, thus making its treatment questionable; as an example, during infections it acts as a defense mechanism to contrast the growth of the invading germs so that the current guidelines do not recommend to lower it [3]. Yet the issue is far from clear: as an example, whereas some studies demonstrated a better short-term outcome in febrile septic shock patients in whom normothermia was obtained with physical cooling, other investigators demonstrated the opposite in patients with bacterial meningitis [4]. Thus, so far, the treatment of fever in patients with documented infections or sepsis is a matter of fierce debate $[5,6]$. These consideration do not apply to heat stroke (HS) in which the very increase of BT represents a life-threatening condition. Independently from the cause(s), its occurrence can be ascribed either (a) to a strenuous physical effort in conditions preventing the dissipation of the excess heat generated (exertional HS - eHs); or (b) to conditions other than exercise, including extremes of age, isolating clothes, the assumption of illicit drug such as cocaine, amphetamines and their derivates, the presence of underlying infections or other medical conditions and the treatment with drugs with anticholinergic properties which reduce the heat loss associated with sweating and evaporation thus hampering the normal compensatory mechanisms. (classic HS-cHS). Environmental factors, such as elevated air temperature and humidity, are fundamental in the pathogenesis of both forms.

The clinical picture associated with HS is determined by the production and release from the immune cells of a number of inflammatory and procoagulative mediators which are involved also in the pathogenesis of septic shock and subsequent Multiple Organ Dysfunction Syndrome (MODS) [7]. Hence, the clinical phenotype of patients with either CHS HS is indistinguishable from that related to septic shock; this similarity is particularly relevant, as it carries the risk of delaying its correct treatment basically consisting in the rapid cooling of the patients and not in the prompt administration of broadspectrum antibiotics $[3,7,8]$.

Throughout July and August 2015 a heat wave (HW) occurred in North-East Italy; in this timeframe a group of patients elevated BT and MODS were admitted to our ICU with the initial diagnosis of septic shock-associated MODS and were treated accordingly; however, the subsequent clinical course indicated that this initial diagnosis was wrong and that the MODS could have been ascribed more appropriately to a HS.

${ }^{\star}$ Correspondence to: Giorgio Berlot, Dept. of Anaesthesia and Intensive Care, Cattinara Hospital, Strada di Fiume 447, 34149 Trieste, Italy, Tel: +390403994540; Fax: +39040912278; E-mail: berlot@inwind.it

Key words: heat stroke, multiple organ dysfunction syndrome, fever, sepsis, septic shock, heat wave

Received: June 26, 2018; Accepted: July 10, 2018; Published: July 13, 2018 


\section{Patients and Methods}

All patients admitted to our ICU with the diagnosis of HS throughout July and August 2015 were enrolled in this study. As the study was retrospective and did not interfere with the standard of care, the approval of the local ethical committee was deemed unnecessary.

The meteorological data were retrieved from a dedicated site (http://www.eurometeo.com); according to the criteria proposed by Bouchama, et al. [8] a HW was defined as three or more consecutive days with the peak air temperature $\geq 32.2^{\circ} \mathrm{C}$ and the diagnosis of HS was established in the presence of an altered mental status and core BT $\geq 40{ }^{\circ} \mathrm{C}$ associated with hot and dry skin. The BT was measured with an ear probe in the admitting ward and via a rectal probe after the ICU admission. The neurologic impairment was evaluated with the Glasgow Coma Scale (GCS) both at hospital and ICU admission and the severity of clinical conditions at ICU admission was measured with the SAPS II score; the individual burden of the concomitant chronic condition(s) and of the underlying frailties were evaluated with the Charlson's index [9] and the CSHA Clinical Frailty Scale [10], respectively. Briefly, the Charlson's index includes nineteen pathologies whose score is calculated according to their impact on mortality and other outcomes, including disability, hospital readmission and length of stay in the hospital: a score $\geq 5$ indicates a relevant impairment of the clinical conditions. The CSHA Clinical Frailty Scale, which can be considered as an expression of the overall functional reserve, ranges from 1 (very fit) to 9 (approaching to the end of life which is expected within 6 months), with intermediate scores indicating a progressive limitation of the everyday activities and/or the need of help.

Other relevant information included the duration of symptoms before hospital admission, the BT measured at the hospital and ICU admission, the medical history, the assumption of drugs in the month preceding the current admission, the blood chemistries, the microbiological data, the interval elapsing from the hospital and the ICU admission and the methods and timing of cooling. In all patients a spinal tap and a brain CT scan were obtained in order to diagnose a cerebro-meningeal infection due to the association of elevated BT with the occurrence of acute neurological disturbances.

\section{Results}

In Trieste, a 220.000-inhabitants town located in North-East Italy, the months of July and August 2015 have been hotter and wetter than in the previous two years (Figure 1). The HW started since the first days of the month; around July the 8th, the temperature slightly decreased for a few days but another rise was observed after July the 12th; after this date, air temperature and humidity remained elevated till August the 16th when the HW ultimately subsided. The patient's admission occurred during the two phases characterized by the hottest temperatures (Figure 2). In the preceding and following years no patient with suspected or confirmed cHS was admitted to our ICU.

Overall, eight patients ( $4 \mathrm{M}, 4 \mathrm{~F}$, age 76 years, IQR $49-81$ years) were diagnosed suffering from cHS and one from eHS $(\mathrm{M}, 22)$ in the considered period (Table 1). According to the informations obtained directly or from the next of kins, all patients with cHS presented comorbidities which in some cases were relevant enough to determine a substantial frailty and none of them patient had access to air conditioning in their homes; moreover, an elevated BT was present for at least 24 hours before hospital admission. Five patients were chronically treated with psychiatric drugs known to be associated with the occurrence of cHS. In all cHS patients initially they were admitted to regular wards and subsequently transferred to the ICU after a variable interval of time primarily due to the deterioration of the neurologic conditions likely due to the failed control of BT (Table 2). In no case the diagnosis of cHS was suspected from the beginning and in the admitting ward the elevated BT was ascribed to an infection and consequently treated with iv. broad-spectrum antibiotics, pending the results of the cultures; a brain CT scan and a sample of the cephalospinal fluid (CSF) were obtained at admission in comatose patients or later on when the neurological conditions worsened; they resulted always negative for cerebro-meningeal infections.

At ICU admission, blood cultures resulted negative in 3 patients and positive in the other ones; in 2 patients of this latter group, however, the cultured germs were considered contaminating (Table 3 ). The diagnosis of septic shock was correct only in the patient n.1 in whom the blood cultures were positive for Streptococcus pyogenes. The patient died few hours after the ICU admission and the likely source of the bloodstream infection was considered a necrotizing pneumonia discovered at the autopsy. In all patients, a target temperature of $\leq 39^{\circ} \mathrm{C}$ was obtained within 12 hours from ICU admission; six patients were treated with the iv. administration of cold fluids whereas in the remaining an intravascular cooling device was used (CoolGard $3000^{\circ}$ and Cool Line $^{\mathrm{Tm}}$, Alsius, Irvine, CA USA); this technique was not available on the other cases. At ICU admission the presence of marked abnormalities of blood chemistries indicated a severe involvement of the kidney and the liver associated with rhabdomyolysis was present (Table 4). Overall, 6 patients (75\%) died in ICU due to unresolving MODS. The autopsy was obtained in three patients. A multifocal myocardial necrosis involving both ventricles was present in two patients and was considered as the expression of a prolonged and sustained exposition to both endogenous and exogenous catecholamines and not a specific marker of HS; pulmonary thromboembolisms were present in all cases, and likely constituted the final cause of death in two of them.

The only patient with eHS was an infantryman who was engaged in a outdoor 8-hour-long drill on the day preceding the hospital admission; six hours after returning to the barracks he presented an elevated BT $41.5^{\circ} \mathrm{C}$ ) associated with confusion; these symptoms were attributed to a meningitis and he was immediately carried to the hospital; as the CT scan and the CSF resulted normal the diagnosis of eHS was hypothesized and he was immediately admitted to the ICU where he received iv cold fluids; after 12 hours his BT returned normal, the state of consciousness was entirely restored and he was discharged from the ICU 2 days later.

\section{Discussion}

The occurrence of HS could be considered as the effect of the interaction of a number of triggering mechanisms able either to induce an increase of BT, such as infections, physical exercise, side effects of some medications and an unusually hot environment impeding its dissipation. Independently from the causes, HS represents a lifethreatening condition requiring a prompt diagnosis and treatment because, once established, the uncontrolled raise of the BT triggers a cascade of events that can lead to the development of MODS and eventually death. The responsible mechanisms are similar to those acting in severe sepsis and septic shock: in a group of critically ill patients with cHS, Huisse, et al. [11] demonstrated elevated blood levels of interleukin (IL)-6, IL-8, IL-1 receptor antagonist (IL1-ra), heat shock proteins 60 and 70 in association with the activation of leukocyte and of the coagulative cascade, in some cases leading to of an overtablet DIC. Unfortunately, as the symptoms of HS overlap with those of sepsis 
Table 1. Patients' characteristics

\begin{tabular}{|c|c|c|c|c|c|}
\hline Pat. n. / sex / age & Heatstroke Type & Comorbidities & Charlson's Index & Frailty Index & Drugs \\
\hline $1 / \mathrm{m} / 49$ & classic & Chronic psychosis & 1 & 5 & lithium \\
\hline $2 / f / 82$ & classic & Depression & 6 & 6 & selergiline \\
\hline $3 / \mathrm{m} / 59$ & classic & Alcoholism & 1 & 3 & tiapride \\
\hline $4 / \mathrm{m} / 73$ & classic & $\begin{array}{l}\text { Arterial } \\
\text { hypertension }\end{array}$ & 5 & 3 & ramipril, oral nitrates \\
\hline $5 / f / 83$ & classic & Ischemic cardiopathy & 4 & 5 & $\begin{array}{l}\text { losartan, ramipril, oral } \\
\text { nitrates }\end{array}$ \\
\hline $6 / f / 80$ & classic & Depression & 2 & 7 & paroxetine \\
\hline $7 / \mathrm{m} / 79$ & classic & Arterial hypertension & 4 & 4 & bisoprolol \\
\hline $8 / \mathrm{f} / 79$ & classic & Parkinson's disease & 1 & 6 & $\begin{array}{l}\text { L-dopa, pramipexol, } \\
\text { sertraline }\end{array}$ \\
\hline $9 / \mathrm{m} / 22$ & exertional & None & 1 & 1 & none \\
\hline
\end{tabular}

Table 2. Relevant clinical variables at Hospital $\left(\mathrm{T}^{\circ} 1\right)$ and ICU $\left(\mathrm{T}^{\circ} 2\right)$ admission

\begin{tabular}{|c|c|c|c|c|c|c|c|c|}
\hline Pat. N. & \begin{tabular}{|c|} 
Duration of \\
symptoms before \\
hospital admission \\
(hours)
\end{tabular} & $\underset{\text { GCS }}{\mathbf{t 1}}$ & $\mathrm{T}_{1}^{\circ}$ & $\operatorname{LOS}_{\text {pre }}$ (hours) & $\begin{array}{c}\text { t2 } \\
\text { GCS }\end{array}$ & $\mathbf{T}_{2}^{\circ}$ & $\begin{array}{c}\text { SAPS II } \\
\text { Score }\end{array}$ & Outcome \\
\hline 1 & 72 & 15 & 40.0 & 24 & 6 & 40.3 & 79 & dead \\
\hline 2 & 72 & 14 & 39.9 & 24 & 3 & 40.3 & 56 & dead \\
\hline 3 & 72 & 9 & 41.4 & 24 & 10 & 39.4 & 72 & discharged \\
\hline 4 & 48 & 14 & 38.8 & 96 & 9 & 41.4 & 105 & dead \\
\hline 5 & 12 & 7 & 41.3 & 0 & 7 & 41.0 & 80 & dead \\
\hline 6 & 24 & 7 & 40.6 & 24 & 7 & 40.9 & 73 & dead \\
\hline 7 & 24 & 15 & 41.0 & 8 & 3 & 41.0 & 92 & dead \\
\hline 8 & 6 & 12 & 42.0 & 48 & 10 & 42 & 42 & discharged \\
\hline 9 & 6 & 7 & 41.5 & 2 & 7 & 41.0 & 50 & discharged \\
\hline
\end{tabular}

Table 3. Microbiological findings and other markers of infections

\begin{tabular}{|c|c|c|c|c|c|}
\hline Pat.N. & WBC $(* 1000 / \mathrm{ml})$ & CRP (mg/dl) & PCT ng/ml & Blood Cultures & Diagnosis of Infection \\
\hline 1 & 29.94 & 373.6 & 439.3 & Str. pyogenes & Confirmed \\
\hline 2 & 8.94 & 15.3 & 81.9 & Negative & Negative \\
\hline 3 & 5.25 & 17.3 & n.a. & Staph. epidermidis & Probably negative \\
\hline 4 & 12.44 & 1.8 & n.a. & Negative & Negative \\
\hline 5 & 14.39 & 0.5 & 43.7 & Staph. epidermidis & Probably negative \\
\hline 6 & 16.69 & 100 & 116 & Staph. epidermidis & Confirmed \\
\hline 7 & 17.31 & 169.2 & 169.2 & Staph. epidermidis & Confirmed \\
\hline 8 & 12.90 & 10.8 & n.a. & Negative & Negative \\
\hline 9 & 15.70 & n.a. & n.a. & Negative & Negative \\
\hline
\end{tabular}

Legend (normal values): n.a.: not available; WBC: white blood cells (4000-11.000 / ml); CRP: C reactive protein ( $<5 \mathrm{mg} / \mathrm{dl})$; PCT: Procalcitonin $(0,1-0,5 \mathrm{ng} / \mathrm{ml})$.

Table 4. Indicators of organ damage within 24 hours after ICU admission

\begin{tabular}{|c|c|c|c|c|c|c|c|}
\hline Pat. & INRr & APTTr & AST/ALT (U.I.) & Creat. (mg/dl) & CPK (U/I.) & TPN I (meg/ml) & LAC (mg/dl) \\
\hline 1 & 1.73 & 1.13 & $73 / 39$ & 5.16 & n.a. & 0.08 & 56.6 \\
\hline 2 & 1.60 & 1.52 & $170 / 53$ & 2.88 & 5234 & 0.57 & 8.8 \\
\hline 3 & 1.78 & 1.16 & $84 / 33$ & 0.83 & 2650 & 0.04 & 19.9 \\
\hline 4 & 2.15 & 0.91 & $168 / 84$ & 2.0 & 430 & 1.4 & 32.5 \\
\hline 5 & 2.08 & 1.15 & $321 / 239$ & 1.2 & n.a. & 0.54 & 40.7 \\
\hline 6 & 3.51 & 1.22 & $4288 / 951$ & 2.86 & n.a. & 22.26 & 57.0 \\
\hline 7 & 1.43 & 0.97 & $49 / 29$ & 1.83 & 604 & 2.29 & 21.9 \\
\hline 8 & 1.59 & 1.10 & $38 / 31$ & 1.0 & 423 & 2.26 & 23.2 \\
\hline 9 & 1.13 & 1.33 & $125 / 84$ & 2.3 & 3733 & 0.27 & 54.2 \\
\hline
\end{tabular}

Legend (normal values): n.a.: not available; INR: international normalized ratio ( 0,78-1,18); APTTr: activated partial thromboplastin time ratio ( $0,89-1,24)$; AST: aspartate transaminase (AST) and alanine transaminase (ALT) $(<45 \mathrm{U} / \mathrm{L})$; creat: creatinine $(0,5-1,3 \mathrm{mg} / \mathrm{dl})$; CPK: creatine phosphokinase $(25-195 \mathrm{U} / \mathrm{L})$; TPN I: Troponin I $(<0,06 \mathrm{mcg} / \mathrm{ml}) ; \mathrm{LAC}$ : Lactate $(5,4-$ $16,2 \mathrm{mg} / \mathrm{dl})$. 


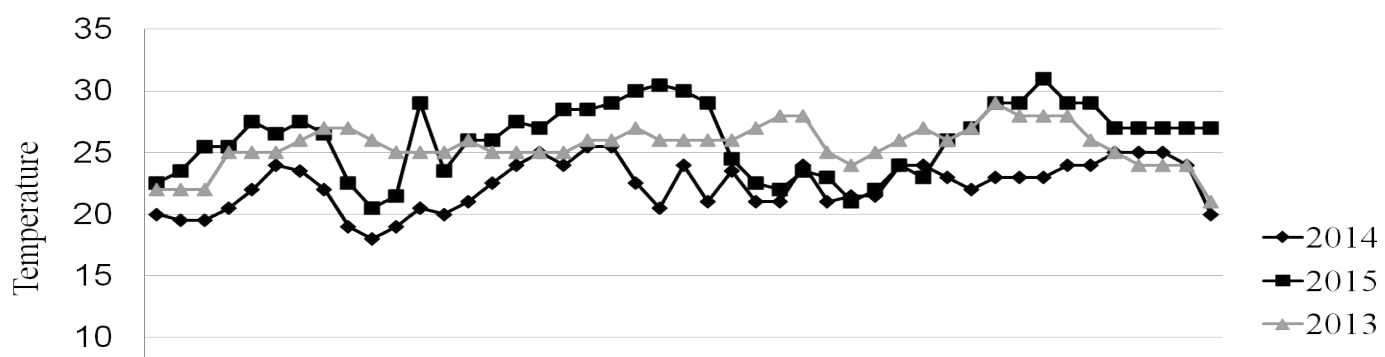

5

0

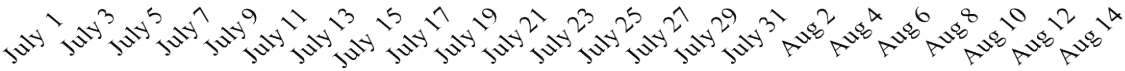

Figure 1. Average temperatures $\left(\mathrm{C}^{\circ}\right)$ during the interval July, the 1st- August, the 15 th in three different years

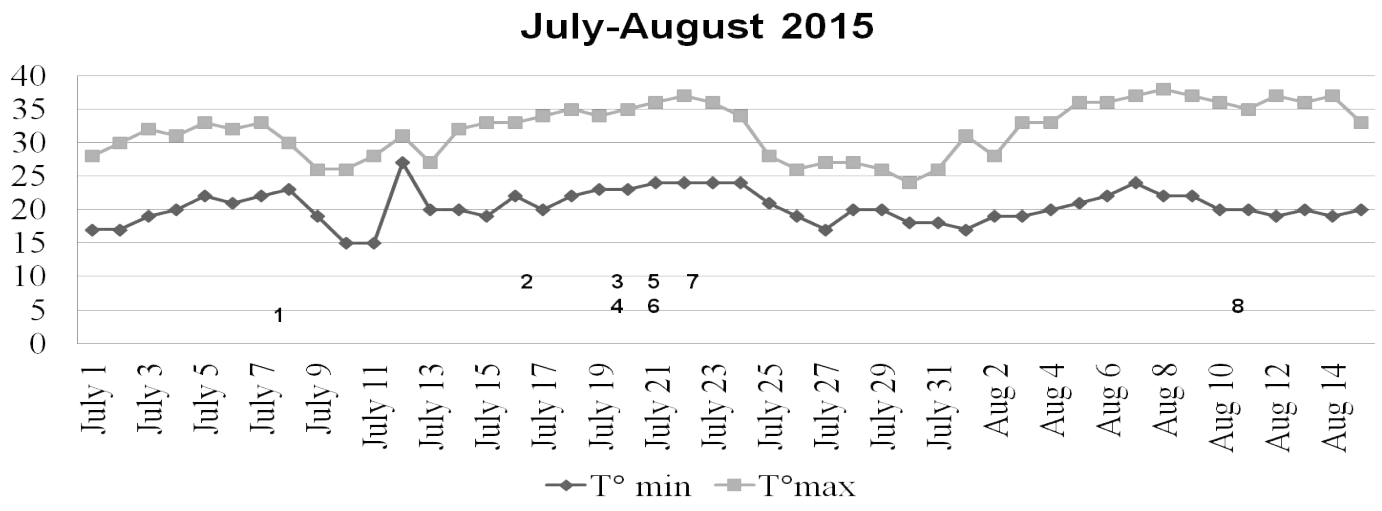

Figure 2. Maximal end minimal temperatures $\left({ }^{\circ} \mathrm{C}\right)$ during the interval July, the 1 st - August, the 15 th. Numbers in the graph indicate the patients

and septic shock, both the correct diagnosis the rapid decrease of BT, which represents the cornerstone of the treatment, can be delayed. This particularly applies to patients suffering from cHS, as the eHS can be hypothesized simply on the basis of the recent history. Thus it appears that in most of cases the diagnosis of HS is one of exclusion which can be particularly challenging where HWs and the related complications are not frequently encountered such as in North-East Italy. Actually, patients with elevated BT and signs of organ dysfunction including a reduced consciousness admitted to the regular wards as well as to the ICU are usually diagnosed as being in sepsis or septic shock possibly associated with meningitis and are given broad-spectrum antibiotics; in these conditions, fever control is not a priority and, potentially, it could even represent a contraindication: recently Young, et al. [12] demonstrated that in infected patients a $\mathrm{BT}$ ranging $39-39,4{ }^{\circ} \mathrm{C}$ in the first 24 hours of admission was associated with a considerable reduction of the risk of death as compared with patients whose BT ranged 36.5$36.9{ }^{\circ} \mathrm{C}$. Moreover, in critically ill patients a number of causes other than infections can determine a MODS associated with a persistently elevated BT, including tumors, drug reactions, autoimmune and connective disorders, transfusion reactions etc. [1,2]. Dissimilarly from HS, in sepsis as well as in all these circumstances the elevated BT is considered more a symptom than a pathologic mechanism in and by itself, and its reduction is not uniformly pursued in the absence of concomitant acute neurologic disorders and/or signs of heart failure.

In our patients, despite the presence of a documented infection in some cases, a number of findings could have suggested the diagnosis of cHS instead of severe sepsis or septic shock. First, all patients were admitted to the hospital after two or three days during which the maximal air temperature largely exceeded the threshold value of 32.2 ${ }^{\circ} \mathrm{C}$ and presented fever unresponsive to antipyretic agents in the days or hours immediately preceding the initial admission; second, most of them had some comorbidities associated with a substantial limitation of everyday activities which likely prevented them from adopting measures able to reduce the environmental heat and humidity; moreover, none of them took advantage from an air conditioning system in their houses; third, the BT was higher than usually observed patients with either infectious or non-infectious diseases; in all these circumstances, the BT usually does not exceed $39^{\circ} \mathrm{C}[13,14]$; actually, in a recent survey only $7.4 \%$ of critically ill adult patients presented a BT $>39.5^{\circ} \mathrm{C}$ [14]; fourth, the presence of an underlying infection should not exclude a cHS: the rate of documented infections in our patients is close to that reported by Hausfater, et al. [15], who recorded a $45 \%$ of documented infections in their patients admitted with cHS during the French HW in 2003; fifth, the clinical course differed from that commonly observed in subjects with severe sepsis and septic shock, in which the early neurologic impairment is not common and the clinical course of MODS is more gradual [16-18]: in our patients a fast deterioration of virtually all organ functions occurred in the few hours preceding the ICU admission. Finally, the increase of the blood levels of CRP and PCT which was observed also in non-infected patients likely reflects the non-septic activation of the inflammatory response, and thus does not help to distinguish septic patients from those with cHS 
[15]. The mortality observed in our patients exceeded that reported in other studies, ranging between $45 \%$ and $65 \%$ [15,19-21]. This could be likely due either to (a) not-modifiable factors, including a relatively more advanced age, the presence of comorbidities and a moderateto-severe decrease of the functional reserve as indicated by the Frailty Index; and (b) to the missed diagnosis of cHS at the hospital admission, with the subsequent delay of the appropriate treatment: this appears to be particularly relevant because, as hypothesized by other investigators $[20,21]$ either the severity of the BT increase and its duration could be associated with the outcome, making mandatory the rapid cooling of the patients. Not surprisingly, the only patient with eHS recovered promptly due to a number of factors, including the younger age, the absence of comorbidities and the shorter interval elapsing from the hospital admission and the initiation of the cooling procedure.

The post-mortem findings of patients died due to cHS are not specific and reflect the prolonged exposure to the elevated levels of endogenous and exogenous catecholamines associated to both the stress response and the ICU treatment [22]. The presence of a pulmonary thromboembolism in all our deceased patients is puzzling: we guess that it could be ascribed to a number of factors, including the pro-coagulative state induced by the elevated body temperature and by the release of sepsis mediators triggering the coagulative cascade [22]; other authors reported a high rate of pulmonary fat embolization in patients died after heat exposure and attributed this findings to fat droplets escaping from the microvascular network disrupted by inflammation [23].

This study has some limitations. First, the cHS was diagnosed in a small number of cases but is likely that some other patients could have suffered from this disorder without being referred to our ICU. Second, it takes into consideration only a limited area but it is possible that many other patients suffered from cHS on a nationwide basis, as an abnormal peak of mortality has been reported throughout Italy by the National Institute of Statistics (ISTAT) during the very same timeframe of our observation and has been attributed to the unusually long-lasting HW [24].

\section{Conclusions}

The diagnosis of HS is elusive as its symptoms close resemble those commonly encountered in severe sepsis and septic shock; this is particularly harmful since the aggressive treatment of fever in these latter conditions is not considered a priority as in HS. An abnormal clustering of patients admitted with the same symptoms, including elevated BT and rapidly evolving MODS, should trigger a more intensive treatment of fever, pending other investigation aimed to confirm or exclude an underlying infection, which, on the other hand, could be the trigger of HS.

\section{References}

1. Marik PE (2000) Fever in the ICU. Chest 117: 855-869.
2. Walter EJm Hanna-Jumma S, Carraretto M, Forni 1 (2016) The pathophysiological basis and consequences of fever. Crit Care 20: 200-210. [Crossref]

3. Rhodes A, Evans LE, Alhazzani W, Lewy MM, Opal SM, et al. (2017) Surviving sepsis campaign: International guidelines for the management of severe sepsis and septic shock: 2016. Intens Care Med 43: 304-377.

4. Taccone FS, Saxena M, Schortgen F (2014) What's new with fever control in ICU. Intensive Care Med 40: 1147-1150.

5. Drewry AM, Hotchkiss RS (2013) Counterpoint: Should antipyretic therapy be given routinely to febrile patients in septic shock? No. CHEST 144: 1098-1100.

6. Mohr NM, Doerschung KC (2013) Point: Should antipyretic therapy be given routinely to febrile patients in septic shock? Yes. CHEST 144: 1096-1097. [Crossref]

7. Hifumi T, Kondo Y, Smimizu K, Miyake Y (2018) Heat Stroke. J of Intensive Care 6: $30-38$

8. Bouchama A, Knochel JP (2002) Heat stroke. N Engl J Med 346: 1978-1988.

9. Quach S, Hennessy DA, Faris P, Fong A, Quan H, Doig C (2009) A comparison between APACHE II and Charlson Index Score for predicting hospital mortality in critically ill patients. BMC Health Serv Res 9: 129. [Crossref]

10. Bagshaw SM, Stelfox HT, McDermid RC, Rolfson DS, Tsuyuki KT, et al. (2014) Association between frailty and short and long-term outcomes among critically ill patient: a multicentre prospective cohort study. CMAJ 186: E95-E102.

11. Huisse M, Pease S, Hurtado-Nedelec M, Arnaud B, Malaquin C, et al (2008) Leukocyte activation: the link between inflammation and coagulation during heatstroke. A study of patients during the 2003 heat wave in Paris. Crit Care Med 36: 2288-2295. [Crossref]

12. Young PJ, Saxena M, Beasley R, Bellomo R, Bailey M, Pilcher D et al (2012) Early peak temperature and mortality in critically ill patients with or without infection. Intensive Care Med 38: 437-444.

13. Marik PE (2014) Early Management of Severe Sepsis Concepts and Controversies. Chest 145: 1407-1418. [Crossref]

14. Niven DJ, Stelfox HT, Shahpori R, Laupland KB (2013) Fever in Adult ICUs (2013) An Interrupted Time Series Analysis. Crit Care Med 41: 1863-1869.

15. Hausfater P, Hurtado M, Pease S, Juillien G, Lvovschi V-E, et al. (2008) Is procalcitonin a marker of critical illness in heatstroke? Intensive Care Med 34: 1377-1383.

16. Vincent J-L, de Mendonca A, Cantraine F, Moreno M, Takala J, et al. (1998) Use of the SOFA score to assess the incidence of organ dysfunction/failure in intensive care units: Results of a multicentre, prospective study. Crit Care Med 26: 1793-1800.

17. Vincent JL, Nelson DR, Williams MD (2011) Is worsening multiple organ failure the cause of death in patients with severe sepsis? Crit Care Med 39: 1050-1055.

18. Doig CJ, Zygun D, Fick GH, Laupland KB, Boiteau PJ, et al. (2004) Study of clinical course of organ dysfuction in intensive care. Crit Care Med 32: 384-390.

19. Pease S, Boudama L, Kermarrec N, Schortgen F, Régnier B, et al. (2009) Early organ dysfunction course, cooling time and outcome in classic heatstroke. Intensive Care Med 35: 1454-1458.

20. Misset B, De Jonghe B, Bastuji-Garin, Gattolliat O, Boughrara E, et al. (2006) Crit Care Med 34: 1087-1092.

21. Herbst J, Mason K, Byard RW, Gilbert JD, Charlwood C, et al. (2014) Heat-related deaths in Adelaide, South Australia: review of the literature and case findings-an Australian perspective. J Forensic Leg Med 22: 73-78. [Crossref]

22. Inoue H, Ikeda N, Tsuji A, Hanagama M, Nata M (2009) Pulmonary fat embolization as a diagnostic finding for heat exposure. Int J Legal Med 11: 1-3.

23. http://www.istat.it/it/files/2015/12/Nota_decessi2015.pdf

Copyright: (C2018 Berlot G. This is an open-access article distributed under the terms of the Creative Commons Attribution License, which permits unrestricted use, distribution, and reproduction in any medium, provided the original author and source are credited. 